\title{
Untersuchungen zur Trübung von Human-Plasma
}

\author{
Von M. Gautschi und R. Richterich \\ Chemisches Zentrallabor des Inselspitals Bern
}

(Eingegangen am 2. Juni/9. Dezember 1972)

Es wird ein durch Streulicht bedingter Extinktionswert $E_{a p p}$ definiert, der aus der Differenz zweier Extinktionswerte bei Nah- und Fernmessung bestimmt wurde. Die Extinktionswerte $E_{a p p}$ für trübe Plasmaproben und Latex-Suspensionen wurden im Bereich von $320-550 \mathrm{~nm}$ untersucht. In einem gewissen Wellenlängenbereich läßt sich das Verhalten der Extinktionswerte $E_{a p p}$ als Funktion der Wellenlänge beschreiben: $\log E_{a p p}=-b \log \lambda+C$. Dabei kann an Modellversuchen mit Latex-Suspensionen gezeigt werden, daß $b$ vorwiegend durch die Teilchengröße und $C$ durch die Teilchenkonzentration bestimmt wird. In Analogie zu diesen Resultaten wurden trübe Plasmaproben untersucht, die in bezug auf die Teilchengröße ein Mehrkomponenten-System darstellen. Die mögliche Anwendung solcher Messungen zur raschen differentialdiagnostischen Erfassung primärer Hyperlipidämien wird erwähnt.

\section{Studies on the turbidity of buman plasma}

An extinction value, $E_{a p p}$, is defined for the measurement of light scattering. It is determined from the difference between the two extinction values for near and distant measurements. The extinction values, $E_{\mathrm{ap} p}$, for turbid plasma samples and latex suspensions were investigated in the range $320-550 \mathrm{~nm}$. In a certain wavelength range, the extinction, $E_{a p p}$, is a function of the wavelength: log $E_{a p p}=-b$ $\log \lambda+C$. In model experiments on latex suspensions, it was shown that $b$ is governed mainly by the particle size, and $C$ by the particle concentration. Analogous measurements were made on turbid plasma, which, with respect to particle size, is a multicomponent system. The possible application of such measurements to the rapid differential diagnosis of primary hyperlipaemias is discussed.

Die optischen spektralen Eigenschaften von HumanPlasma sind einerseits durch das Vorliegen bestimmter Farbstoffe (1), andererseits durch Trübung bedingt. Die Farbstoffe sind z. T. bekannt, so etwa HämoglobinDerivate, Bilirubin und Carotinoide und führen $\mathrm{zu}$ Schultern oder Gipfeln bei bestimmten Wellenlängen (Abb. 1). Der kontinuierliche Anstieg der Extinktion

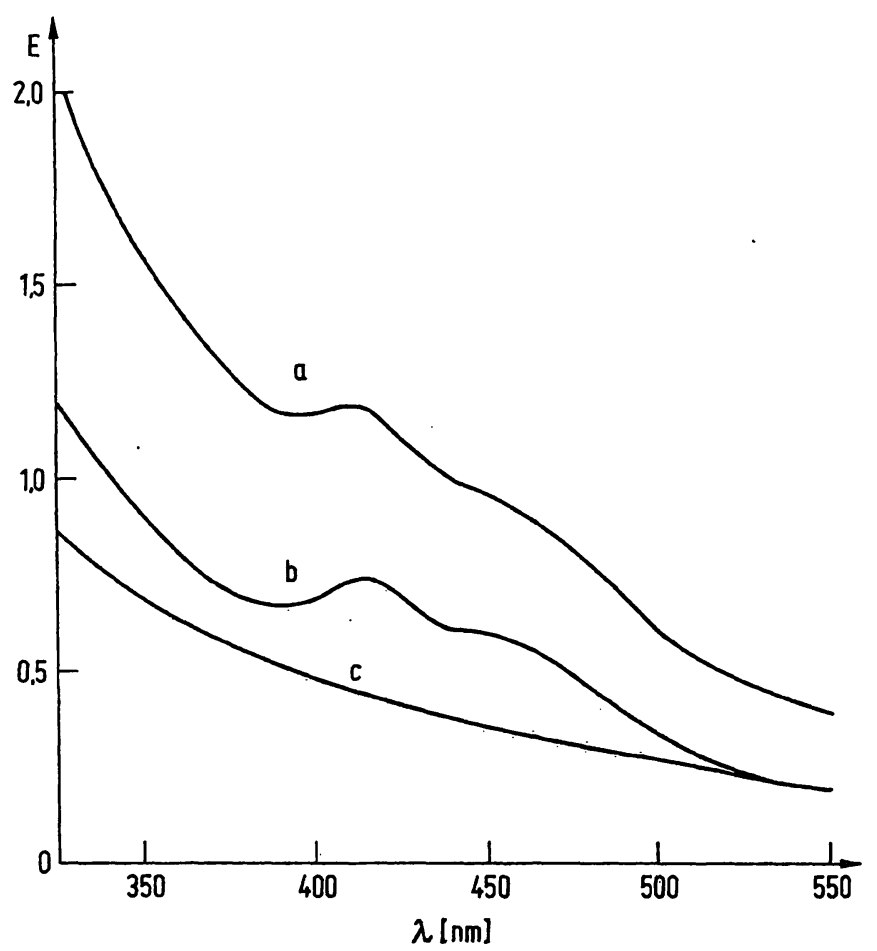

Abb. 1

Spektralkurve einer unverdünnten Plasma-Probe.

a) Messung in Fernstellung, b) Messung in Nahstellung und c) die trübungsbedingte Differenz $\left(E_{\mathrm{app}}\right)$ zwischen $\left.a\right)$ und $\left.b\right)$ vom langwelligen zum kurzwelligen Bereich ist hingegen weitgehend durch Trübung bedingt. Die wichtigsten für diese Trübung verantwortlichen Partikel sind in Tabelle 1 zusammengestellt. Da diese Partikel, vor allem im Zusammenhang mit Fragen des LipidStoffwechsels von großem Interesse sind, stellt sich die Frage, ob sich durch Messung der Trübung von Plasma-Proben möglicherweise Rückschlüsse auf die Größe und Konzentration der Partikel ziehen lassen.

GAEBLER (2) hat im Jahre 1943 offenbar als erster die Beziehung zwischen Wellenlänge und Extinktion bei trüben Hunde-Seren untersucht. Er fand eine lineare Beziehung zwischen den Logarithmen der Extinktion und dem negativen Logarithmus der Wellenlänge im Bereich zwischen 600 und $1000 \mathrm{~nm}$. Fog (3) untersuchte die Trübung verschiedener Modell-Suspensionen und von Human-Plasma zwischen 350 und $1000 \mathrm{~nm}$ und fand die folgende Beziehung:

$$
\mathrm{E}=\mathrm{k} \cdot \lambda^{-\mathrm{y}} \text {, }
$$

oder logarithmiert

$$
\log \mathrm{E}=-\mathrm{y} \log \lambda+\log \mathrm{k},
$$

Tab. 1

Partikel, die im Plasma die Streulichtintensität beeinflussen können

\begin{tabular}{lc}
\hline \multicolumn{1}{c}{ Streuende Partikel } & $\begin{array}{c}\text { Mittlerer } \\
\text { Durchmesser } \\
{[\mu \mathrm{m}]}\end{array}$ \\
\hline Fibrinpartikel, Thrombocyten & 1,0 \\
Chylomikronen & $0,1-0,5$ \\
prä- $\beta$-Lipoproteine & $\mathrm{ev}-1,0$ \\
$\beta$-Lipoprotelne & $0,02-0,07$ \\
Plasma-Proteine & 0,03 \\
\hline
\end{tabular}


wobei $\mathrm{E}$ der Extinktion, $\lambda$ der Wellenlänge und $\mathrm{k}$ einer Konstanten entspricht. NIELSEN (4-6) untersuchte die gleichen Zusammenhänge in Human-Serum, um bei Farbstoff-Analysen mathematisch einen Leerwert ermitteln zu können. Im Prinzip fand er zwischen 600 und $920 \mathrm{~nm}$ eine Bestätigung von „GAeBLER's law"

$$
-\log \mathrm{E}=\mathrm{c} \log \lambda+\mathrm{k}^{*}
$$

darin $k^{*}$ eine Konstante und $c$ eine von der Plasmaprobe abhängige Variable darstellt.

Physikalisch ist durch die RAYLEIGH'sche Gleichung (7) die Streulicht-Intensität $I_{s}$ als Funktion der einfallenden Licht-Intensität $I_{0}$ und der Wellenlänge $\lambda$ wie folgt gegeben (s. Anhang):

$$
\mathrm{I}_{\mathrm{s}}=\mathrm{k} \cdot \mathrm{I}_{0} \frac{1}{\lambda^{4}} .
$$

Die Konstante $\mathrm{k}$ ist dabei durch Größe, Form, Polarisierbarkeit sowie die Meßgeometrie bestimmt. Die RAYLEIGH'sche Gleichung gilt aber nur unter bestimmten Voraussetzungen: Einfach-Streuung und kleiner Partikel-Durchmesser $(d<0,2 \lambda)$. Unter diesen Bedingungen und bei kleinen Extinktions-Werten gilt nach SAurer (8) für die Trübungs-Extinktion $E$ und die Streu-Intensität $I_{s}$ :

$$
E=f \cdot I_{s} .
$$

Nach Einsetzen in die Rayleigh'sche Gleichung ergibt sich:

$$
E=f \cdot k \cdot I_{0} \frac{1}{\lambda^{4}} .
$$

Unter den hier vorliegenden Versuchs-Bedingungen sind $\mathrm{f}, \mathrm{k}$ und $\mathrm{I}_{0}$ Konstanten. Ihr Produkt kann daher in einer neuen Konstanten $\mathrm{K}$ zusammengefaßt werden. Logarithmiert man die Gleichung

$$
\mathrm{E}=\mathrm{K} \frac{1}{\lambda^{4}}
$$

so erhält man:

$$
\log \mathrm{E}=-4 \log \lambda+\log \mathrm{K} .
$$

Diese theoretisch zu erwartende Beziehung stimmt im Prinzip mit den Resultaten der oben erwähnten Untersuchungen überein. Wie jedoch aus dem Folgenden hervorgeht, kommt es sowohl bei den Modell-Versuchen, wie auch bei Human-Plasma durch Einfachund Mehrfach-Streuung an größeren isotropen kugelförmigen Teilchen $(d \geqq 0,2 \lambda)$ zu komplizierteren Verhältnissen, so daß obige Gleichung allgemeiner formuliert werden muß:

$$
\log \mathrm{E}=-\mathrm{b} \log \lambda+\mathrm{C} \text {. }
$$

Dabei entspricht $b$ der Steigung der Geraden $[\log E=f(\log \lambda)]$ und $C$ dem Schnittpunkt der Geraden mit der Ordinate.

In der vorliegenden Arbeit wurde zunächst untersucht, in welchem Wellenlängen-Bereich und unter welchen Meßbedingungen diese Gleichung einer Regressionsgeraden für $\log \mathrm{E}$ und $\log \lambda$ gültig ist. Innerhalb so festgelegter Wellenlängen-Bereiche erfolgten alle $\mathrm{Be}$ stimmungen der Regressionsgeraden für trübe Stan- dard- und Plasmaproben. Modellsuspensionen kugelförmiger Teilchen dienten dazu, den Einfluß der Partikel-Größe, Partikel-Konzentration und Schichtdicke auf die Parameter $b$ und $C$ der Regressionsgeraden zu analysieren. Die gewonnenen Erkenntnisse wurden dann auf Human-Plasma ausgedehnt.

\section{Material und Methoden}

\section{Material}

Für die Modell-Versuche dienten Polystyrol-Latex-Suspensionen kugelförmiger Teilchen, die von der Fa. Serva, Feinbiochemica, Heidelberg, erhalten wurden (Tab. 2). Alle Verdünnungen wurden mit demineralisiettem Wasser durchgeführt. Die VerdünnungsAngaben erfolgten in Vol-\% der Originallatexsuspensionen mit $10 \%$ Trockensubstanż. Bei der Untersuchung der Plasma-Trübung wurden makroskopisch trübe, in der Routine anfallende und nicht besonders ausgewählte Proben verwendet. Verdünnung und Untersuchung erfolgten innerhalb $24 \mathrm{~h}$ nach der Blut-Entnahme. Zur Verdünnung wurde $0,154 \mathrm{~mol} / \mathrm{l}(9 \mathrm{~g} / \mathrm{l})$ Natrium-ChloridLösung verwendet.

\section{Meß-Verfahren}

Alle Messungen wurden mit einem Spektral-Photometer Unicam SP 800 durchgeführt. Um den Einfluß der Schichtdicke auf die trübungs-bedingte Extinktion ( $E_{\mathrm{app}}$, app = apparent) $z \mathrm{u}$ untersuchen, wurden folgende Quarz-Küvetten verwendet: $0,2 \mathrm{~cm}$, $0,5 \mathrm{~cm}$ und $1,0 \mathrm{~cm}$ Schichtdicke. Die Küvette mit der ProbenLösung wird in die Standard-Stellung (Fern-Stellung) des Unicam SP 800 gebracht. Die Extinktions-Differenż gegen Wasser wird registriert. Diese „Fern-Messung“ ergibt eine erste SpektralKurve $K_{1}$ (Abb. 1 und 2). Eine zweite Spektral-Kurve $K_{2}$ wird durch Nah-Messung ermittelt. $Z$ u diesem $Z$ weck wird die Küvette mit der Proben-Lösung in die Nah-Stellung gebracht, wobei die Vergleichs-Lösung (Wasser) in der ursprünglichen Stellung verbleibt. Aus den durch Fern- und Nahmessung erhaltenen SpektralKurven wurden nun in Intervallen von $10 \mathrm{~nm}$ die Differenzen der Extinktionen als Funktion der Wellenlänge ermittelt (Abb. 1 und 2). Die auf diese Weise erhaltenen Extinktionswerte $E_{a p p}$ sind vorwiegend durch Streu-Licht bedingt. Spektrale AbsorptionsEigenschaften (Eigen-Farbe) der Probe-Lösungen werden durch diese Differenz-Bildung kompensiert; dies ist vor allem bei PlasmaProben von Bedeutung.

Die oben definierten und gemessenen Extinktionswerte $E_{\text {app }}$ wurden in die eingangs abgeleitete Funktion,

$$
\log E=-b \log \lambda+C,
$$

eingesetzt und die entsprechenden Regressions-Geraden berechnet. Aus technischen Gründen (zu hohe oder zu tiefe ExtinktionsWerte) mußten die Plasma-Proben für die Berechnung der Regressions-Geraden in zwei Gruppen aufgeteilt werden, damit möglichst bei allen Proben eine gleiche $Z$ ahl von Messungen durchgeführt werden konnte. In der 1 . Gruppe erfolgte die Auswertung zwischen 320 und $500 \mathrm{~nm}$, bei der 2. Gruppe zwischen 350 und $550 \mathrm{~nm}$.

Tab. 2

Charakteristika der verwendeten Latex-Suspensionen

\begin{tabular}{cccc}
$\begin{array}{c}\text { Mittlerer } \\
\text { Durchmesser }\end{array}$ & $\begin{array}{c}\text { Mittlere } \\
\text { Standardab- } \\
\text { weichung des } \\
\text { Durchmessers } \\
\mu \mathrm{m}\end{array}$ & $\begin{array}{c}\text { Mittleres } \\
\text { Partikel- } \\
\text { Volumen }\end{array}$ & $\begin{array}{c}\text { Mittlere } \\
\text { Partikel- } \\
\text { Konzentration }\end{array}$ \\
\hline$\mu \mathrm{m}$ & 0,003 & $6,78 \times 10^{-4}$ & $\mathbf{N} / \mathrm{ml}$ \\
\hline 0,109 & 0,002 & $1,59 \times 10^{-2}$ & $6,10 \cdot 10^{12}$ \\
0,312 & 0,003 & $6,55 \times 10^{-2}$ & $1,46 \cdot 10^{12}$ \\
0,500 & 0,006 & $2,78 \times 10^{-1}$ & $3,42 \cdot 10^{12}$ \\
0,810 & & & \\
\hline
\end{tabular}




\section{Resultate}

\section{Modell-Versuche}

\section{Spektrale Eigenschaften}

In Abbildung 2 ist das Spektrum einer 0,6 Vol- $\%$ Polystyrol-Latex-Lösung (Partikel-Durchmesser 0,1 $\mu \mathrm{m}$, Schicht-Dicke $0,5 \mathrm{~cm}$ ) dargestellt. Aufgezeichnet sind $\mathrm{K}_{0}$, der Wasser-Leerwert, sowie $\mathrm{K}_{1}$, Messung in Fernstellung und $\mathrm{K}_{2}$, Messung in Nah-Stellung. Die Differenz $z$ wischen $K_{1}$ und $K_{2}$ entspricht der scheinbaren Trübung $\mathrm{E}_{\mathrm{app}}$. Ungefähr die Hälfte bis ein Drittel der Gesamt-Extinktion ist bei diesen Suspensionen durch Trübung bedingt.

\section{Beziebung zwischen Partikel-Größe und $\mathrm{E}_{\mathrm{app}}$}

Als erstes wurde $E_{a p p}$ als Funktion der Wellenlänge für verschiedene Teilchen-Größen und Teilchen-Konzentrationen untersucht. Die Resultate dieser GrundVersuche sind in Abbildung 3 zusammengefaßt. Es kamen vier verschiedene Partikel-Größen $(0,1,0,31$, $0,5$ und $0,81 \mu \mathrm{m})$ bei je vier verschiedenen Verdünnungen über einen Bereich von $320-550 \mathrm{~nm}$ zur Untersuchung. Bei den $0,1 \mu \mathrm{m}$ Suspensionen ergab sich über den ganzen Meß-Bereich, unabhängig von der Konzentration, eine lineare Beziehung zwischen dem Logarithmus von $\mathrm{E}_{\text {app }}$ und dem Logarithmus der Wellenlänge. Je größer jedoch die Partikel werden, um so früher flacht sich der lineare Teil gegen das Kurzwellige ab. Diese Abflachung gegen die Abszisse ist bereits bei den $0,31 \mu \mathrm{m}$ Partikel-Suspensionen zu erkennen. Bei den 0,5 $\mu \mathrm{m}$ Suspensionen ist eine Linearität nur noch etwa zwischen 470 und $550 \mathrm{~nm}$ nachweisbar. Bei der $0,8 \mu \mathrm{m}$ Suspension liegt zwischen 370 und $430 \mathrm{~nm}$ praktisch ein Plateau vor. Aus diesen Beobachtungen muß geschlossen werden, daß mit zunehmender Partikel-Größe der Gültigkeitsbereich der eingangs abgeleiteten Formel progredient kleiner wird

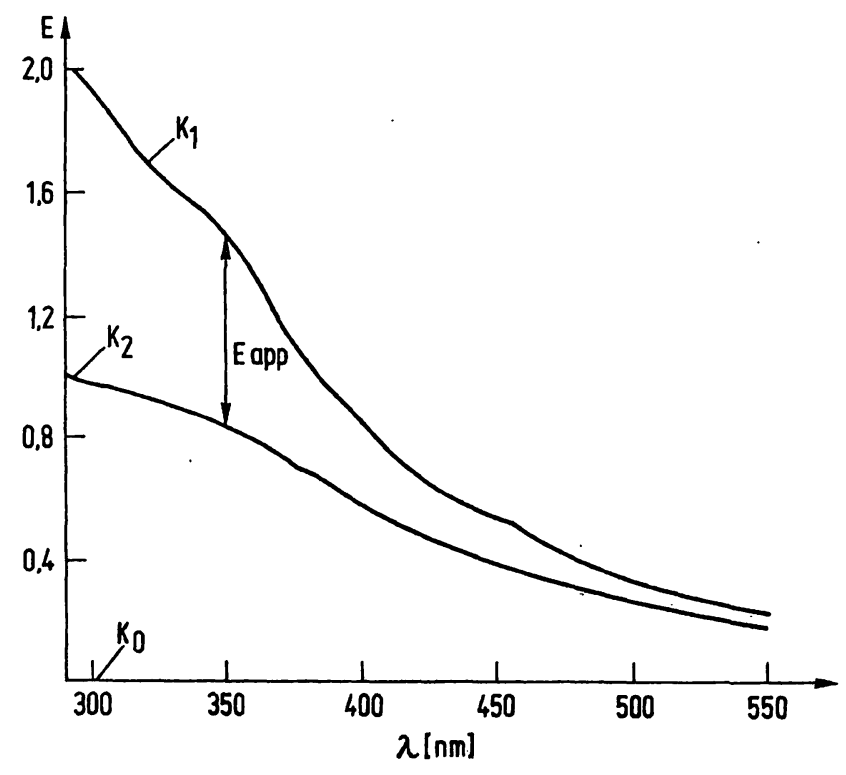

Abb. 2

Spektralkurven einer 0,6 Vol-\% Polystyrol-Latex-Suspension (Partikeldurchmesser $0,1 \mu \mathrm{m}$, Schichtdicke $0,5 \mathrm{~cm}$ ): $\mathrm{K}_{0}$ Leerprobe (Wasser), $K_{1}$ Probe in Fernstellung, $K_{a}$ Probe in Nahstellung, $E_{a p p}$ trübungsbedingte Extinktion

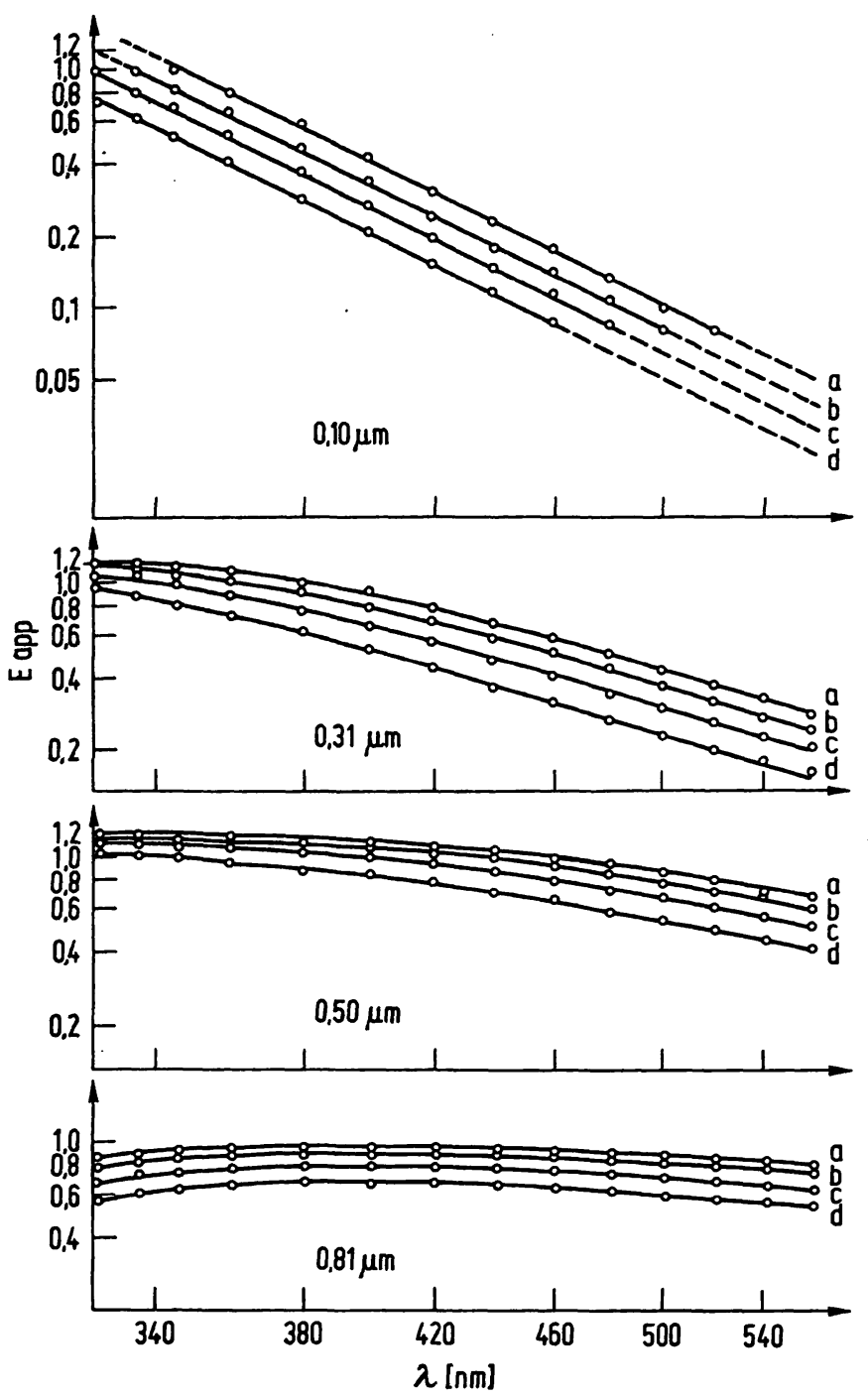

Abb. 3

Beziehung zwischen Wellenlänge und $E_{a p p}$ von verdünnten LatexSuspensionen mit mittleren Partikeldurchmessern von $0,109,0,312$ 0,50 und $0,81 \mu \mathrm{m}$. Kurve a) 0,8 Vol- $\%$, b) 0,7 Vol- $\%$, c) 0,6 Vol- $\%$ und d) 0,5 Vol- $\%$

und sich die untere Grenze der Gültigkeit immer mehr nach dem Langwelligen verschiebt.

\section{Beqiebung qwischen Partikel-Konzentration und $\mathrm{E}_{\mathrm{app}}$}

Aus Abbildung 3 geht ebenfalls hervor, daß nicht nur die Partikel-Größe einen Einfluß auf die RegressionsGerade hat, sondern auch die Partikel-Konzentration. Dieser Einfluß der Partikel-Konzentration im Sinne einer Verlagerung der unteren Grenze des linearen Abschnittes erfolgt gleichsinnig wie derjenige der Partikel-Größe. Am ausgeprägtesten scheint der Einfluß der Partikel-Konzentration bei den 0,31 und $0,5 \mu \mathrm{m}$ Suspensionen.

\section{Beziebung zuvischen Partikel-Größe und b}

Für die Analyse der Parameter, welchen den Regressions-Koeffizienten $b$ beeinflussen, wurden nur jene Kurven-Abschnitte berücksichtigt, bei denen von Auge eine Linearität vermutet wurde. Die Beziehung zwischen Partikel-Durchmesser und $b$ sind in Abbildung 4 und Tabelle 3 zusammenfassend dargestellt. Die Auswertung der in Abbildung 3 dargestellten Daten zeigt 


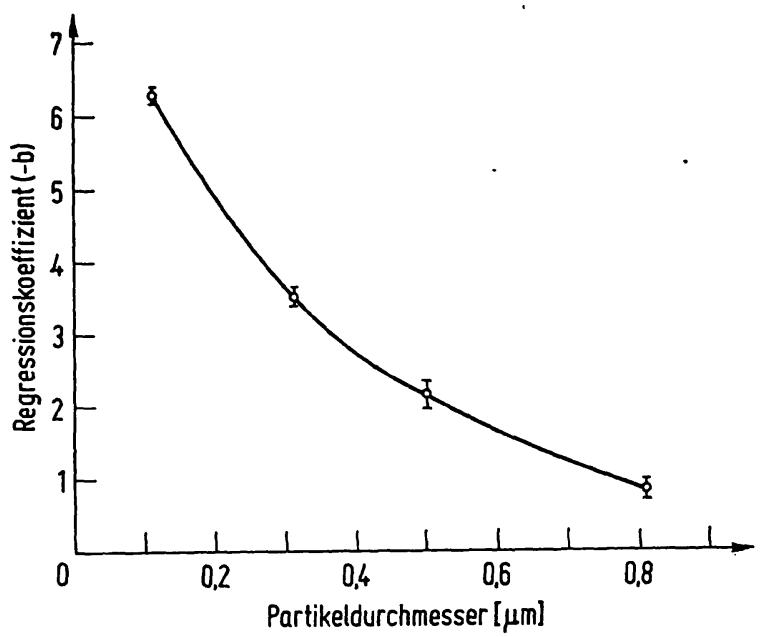

Abb. 4

Mittelwerte der in Tab. 3 für jede Partikelgröße gemessenen Regressionskoeffizienten (b), aufgetragen gegen die Partikeldurchmesser (mit Streubereich)

Tab. 3

Regressionskoeffizienten $b$ und entsprechende Korrelationskoeffizienten $r$ für verschiedene Latexsuspensionen (Auswertung der linearen Kurvenverläufe der Abb. 3; b wurde aus 10 Meßwerten in $10 \mathrm{~nm}$ Abständen von $470-560 \mathrm{~nm}$ ermittelt

\begin{tabular}{ccc}
\hline $\begin{array}{c}\text { Mittlerer Durchmèsser } \\
\text { der Latexpartikel } \\
{[\mu \mathrm{m}]}\end{array}$ & $\mathrm{b}$ & $\mathrm{r}$ \\
\hline 0,1 & $-6,29$ & 0,999 \\
& $-6,38$ & 0,999 \\
& $-6,27$ & 9,999 \\
& $-6,21$ & 0,999 \\
0,3 & $-3,67$ & 0,999 \\
& $-3,67$ & 0,999 \\
& $-3,53$ & 0,999 \\
& $-3,39$ & 0,997 \\
0,5 & $-1,99$ & 0,998 \\
& $-2,12$ & 0,999 \\
& $-2,29$ & 0,999 \\
& $-2,37$ & 0,999 \\
0,8 & $-0,70$ & 0,997 \\
& $-0,73$ & 0,998 \\
& $-0,92$ & 0,998 \\
& $-1,00$ & 0,999 \\
\hline
\end{tabular}

eindeutig, daß die Absolut-Größe des RegressionsKoeffizienten mit zunehmender Partikel-Größe abfällt. Dies läßt den wichtigen Schluß zu, daß jeder Partikel-Größe ein bestimmter Regressions-Koeffizient zugeordnet werden kann. Die Differenzen der AbsolutWerte sind dabei, wie vor allem aus Abbildung 4 hervorgeht, recht bedeutend.

\section{Beqiebung zivischen Partikel-Konzentration und $\mathrm{b}$}

Der Einfluß der Partikel-Konzentration auf den Regressions-Koeffizienten $\mathrm{b}$ ist in Tabelle 3 und $4 \mathrm{zu}-$ sammenfassend dargestellt. Wie daraus hervorgeht, ist bei den kleinen Partikeln keine Beeinflussung nachzuweisen. Bei den größeren Partikeln läßt sich ein Einfluß beobachten, der aber wahrscheinlich dadurch bedingt ist, da $B$ ein Teil des nicht-linearen Bereiches bei der Berechnung einbezogen wurde.

\section{Beqiebung qwischen Scbicht-Dicke und $\mathrm{b}$}

Die Werte des Regressionskoeffizienten b zeigten keine faßbare Abhängigkeit von der variablen Schichtdicke (Tab. 4).

\section{Mebrkomponenten-Systeme und $\mathrm{b}$}

Nachdem bisher gezeigt wurde, daß der RegressionsKoeffizient überwiegend eine Funktion der PartikelGröße ist, stellte sich die Frage nach dem Verhalten

Tab. 4

Regressionskoeffizienten $b$ und Korrelationskoeffizienten $r$ für LatexSuspensionen verschiedener Konzentration (Partikeldurchmesser $0,1 \mu \mathrm{m})$, bei mehreren Schichtdicken gemessen

\begin{tabular}{cccc}
\hline $\begin{array}{c}\text { Schichtdicke } \\
{[\mathrm{cm}]}\end{array}$ & $\begin{array}{c}\text { Konzentration } \\
\text { [Vol. \%] }\end{array}$ & $\mathrm{b}$ & $\mathrm{r}$ \\
\hline 0,2 & 0,8 & $-6,57$ & 0,999 \\
& 0,7 & $-6,46$ & 0,999 \\
& 0,6 & $-6,51$ & 0,998 \\
0,5 & 0,8 & $-6,29$ & 0,999 \\
& 0,7 & $-6,38$ & 0,999 \\
& 0,6 & $-7,27$ & 0,999 \\
& 0,5 & $-6,21$ & 0,999 \\
& 0,4 & $-6,43$ & 0,999 \\
& 0,3 & $-6,32$ & 0,999 \\
1,0 & 0,5 & $-6,75$ & 0,999 \\
& 0,4 & $-6,92$ & 0,999 \\
& 0,3 & $-6,68$ & 0,999 \\
\hline
\end{tabular}

Tab. 5

Regressionskoeffizienten $b$ und Korrelạtionșkoeffizienten $r$ für die linearen Abschnitte der Regressionskurven $A-G$ in Abbildung 5 (aus je 10 Meßwerten in $10 \mathrm{~nm}$ Abständen voń $470=560 \mathrm{~nm}$ ermittelt). $q=$ Quotient aus der Konzentration der ersten Partikelkomponente $(0,5 \mu \mathrm{m}$ Durchmesser) und der Konzientration der zweiten Partikelkomponente $(0,1 \mu \mathrm{m}$ Durchmesser)

\begin{tabular}{cccl}
\hline Regressionskurve & $\mathrm{b}$ & $\mathrm{r}$ & $\mathrm{q}$ \\
\hline A & $-2,20$ & 0,999 & 1,5 \\
B & $-2,42$ & 0,999 & 0,625 \\
C & $-2,72$ & 0,999 & 0,333 \\
D & $-3,03$ & 0,999 & 0,187 \\
E & $-3,21$ & 0,999 & 0,1 \\
F & $-4,27$ & 0,999 & 0,042 \\
G & $-4,89$ & 0,998 & 0,021 \\
\hline
\end{tabular}

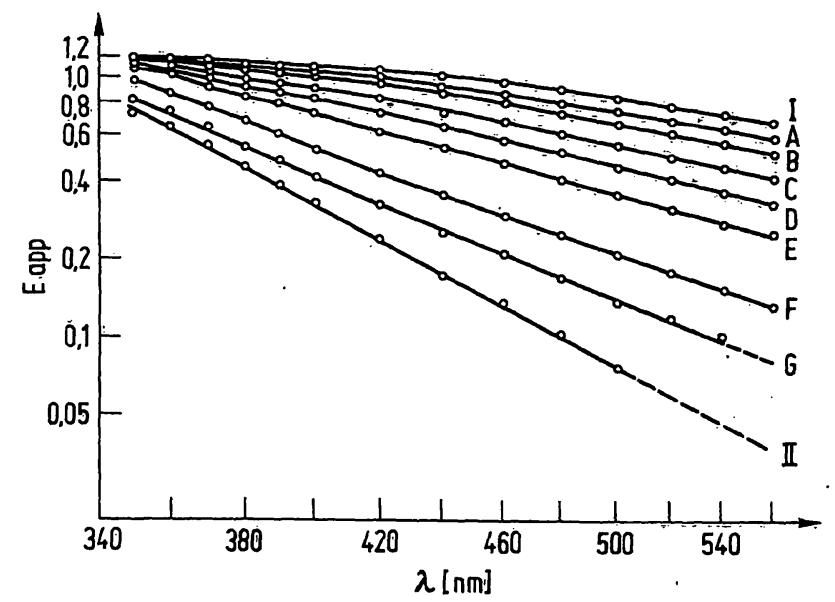

Abb. 5

Regressionskurven von Mischungen zweier verdünnter Latex-Suspensionen mit Partikeldurchmessern von $0,1 \mu \mathrm{m}$ und $0,5 \mu \mathrm{m}$. Kurve I pensionen mit Partikeldurchmessern von $0,1 \mu \mathrm{m}$ und $0,5 \mu \mathrm{m}$. Kurve 0,7 Vol- $\%(0,1 \mu \mathrm{m})$, Partikel Kurven $A-G$, verschiedene Mischungsverhältnisse nach den Angaben in Tab. 5 


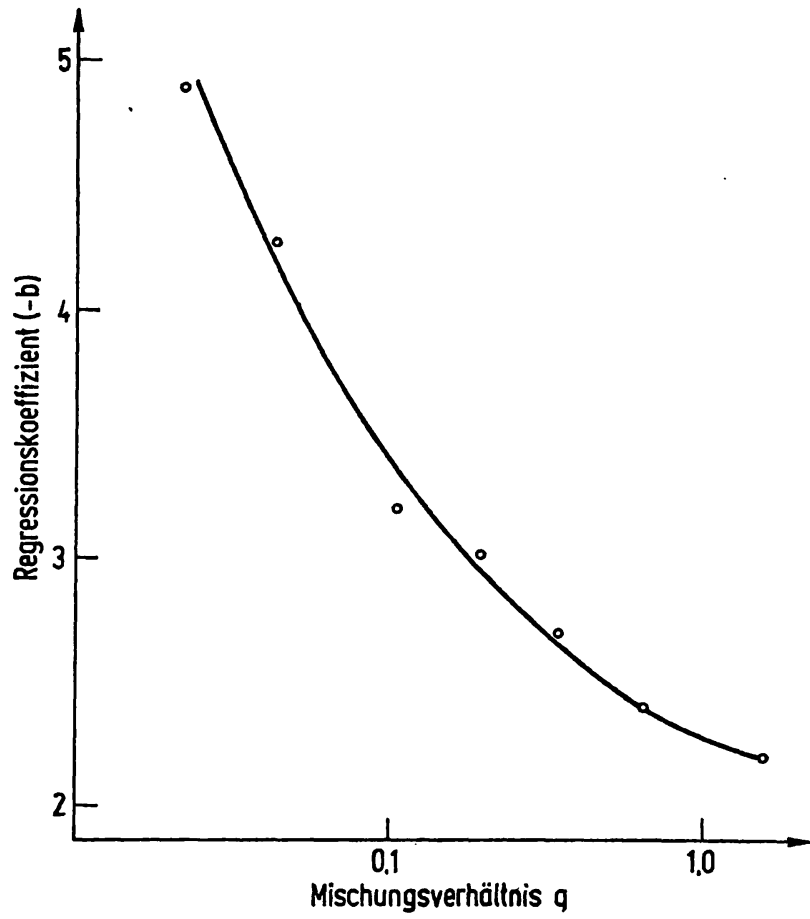

Abb. 6

Regressionskoeffizienten (b) der linearen Abschnitte der Kurven A-G aus Abb. 5 in Abhängigkeit des Mischungsverhältnisses der beiden Partikelkomponenten. q Quotient aus der Konzentration der ersten Komponente $(0,5 \mu \mathrm{m}$ Durchmesser $)$ und der Konzentration der zweiten Komponente $(0,1 \mu \mathrm{m}$ Durchmesser)

eines Gemisches von Partikeln verschiedener Größe. In Tabelle 5 und Abbildung 5 sind die Ergebnisse eines solchen Experimentes dargestellt. Es wurden Suspensionen von $0,1 \mu \mathrm{m}$ und $0,5 \mu \mathrm{m}$ Partikeln gemischt und die Regressions-Geraden und -Koeffizienten berechnet. Wie vor allem aus Abbildung 6 hervorgeht, kann dem Regressions-Koeffizienten einer 2-Komponenten-Suspension ein bestimmter $\mathrm{q}$-Wert (Quotient aus den in einer Latexmischung enthaltener. Konzentrationen zweier Partikelkomponente: 1 ) zugeordnet werden. Bei sehr hohen und sehr tiefen q-Werten nähert sich die Kurve den Regressions-Koeffizienten b, die den einzelnen Ausgangs-Suspensionen entsprechen. Diese Beobachtung ist sehr wichtig, läßt sich doch vermuten, daß die oben ermittelten Gesetzmäßigkeiten auch für heterogene Suspensionen kugelförmiger Partikel gelten.

\section{Parameter, die $\mathrm{E}_{\mathrm{app}}$ beeinflussen}

Während bisher nur der Einfluß verschiedener Variabler auf den Regressions-Koeffizienten untersucht wurde, seien im folgenden die Beziehungen zur scheinbaren Trübung $E_{a p p}$ analysiert. Wie aus Abbildung 7 hervorgeht, ist bei konstanter Wellenlänge, PartikelGröße und Schichtdicke $E_{a p p}$ deutlich von der Konzentration abhängig. Bei Partikeln von $0,1 \mu \mathrm{m}$ liegt dabei keine dem LAMBERT-BEER'schen Gesetz analoge Funktion vor, doch läßt sich eine solche bei größeren Partikeln vermuten (Abb. 8). Bei Änderungen der Schicht-Dicken blieben die $E_{a p p}$ proportional zueinander.

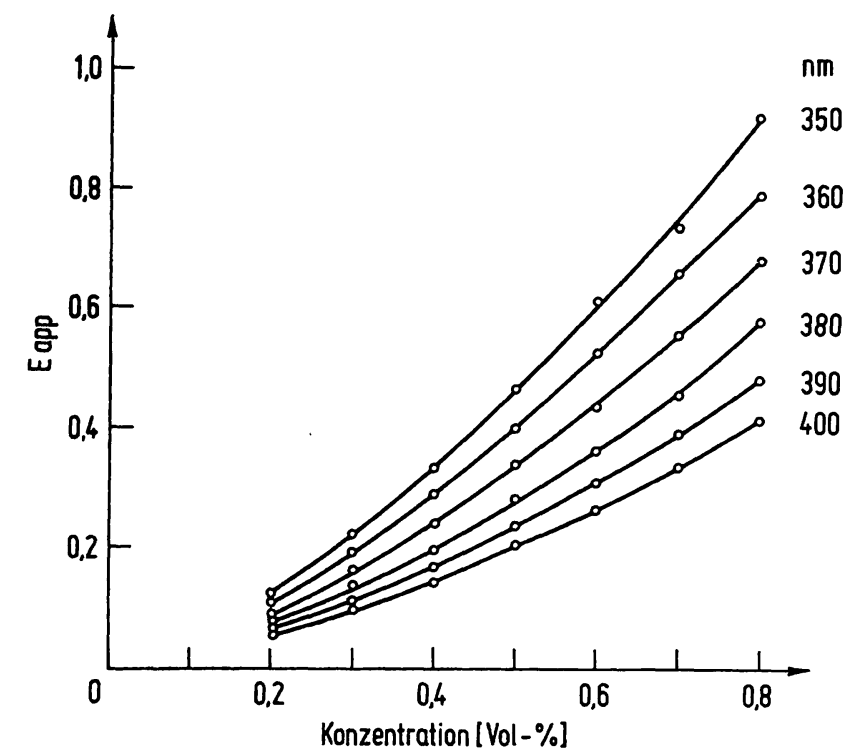

Abb. 7

$E_{\mathrm{app}}$ einer Latex-Verdünnungsreihe als Funktion der Wellenlänge und der Konzentration (Partikeldurchmesser $0,1 \mu \mathrm{m}$ )

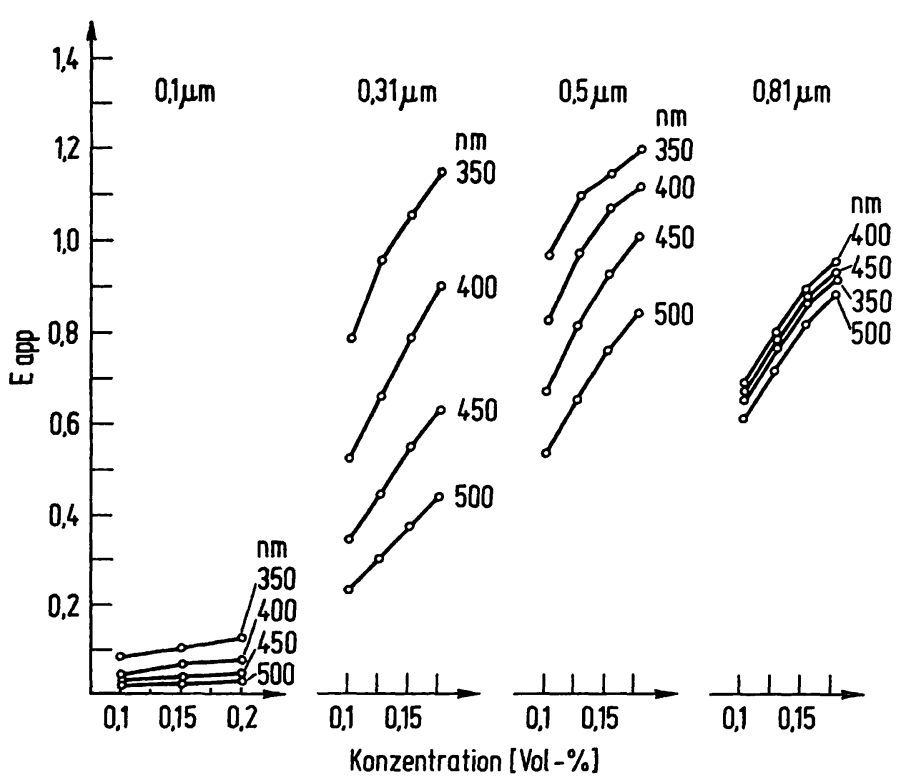

Abb. 8 E gpp verschieden großer Teilchen $(0,1 \mu \mathrm{m}, 0,31 \mu \mathrm{m}, 0,50 \mu \mathrm{m}$ und
$0,81 \mu \mathrm{m})$ als Funktion der Konzentration und der Wellenlänge (Verdünnung in Vol-\% der Originallatexsuspensionen mit $10 \%$ Trockensubstanz)

In Abbildung 8 ist das Verhalten verschieden großer Teilchen bei gleicher Konzentration als Funktion der Wellenlänge dargestellt. Hier ergeben sich nun recht komplizierte Verhältnisse. Bei Partikel-Suspensionen um $0,81 \mu \mathrm{m}$ fanden sich die höchsten $E_{\text {app }}$ bei $400 \mathrm{~nm}$; für dieses Abweichen von der Regel dürfte der oben erwähnte Plateau-Effekt verantwortlich sein. Bei den kleineren Partikeln werden die höchsten $E_{\text {app }}$ im kurzwelligen Bereich beobachtet. Zusammenfassend läßt sich sagen, daß für Teilchen einer bestimmten Größe eine gewisse Beziehung zwischen $E_{a p p}$ und der Konzentration besteht, daß diese Beziehung aber nicht einfacher Natur ist. 
Trübe Plasma-Proben

\section{Spektrale Eigenschaften}

Ein typisches Spektrum eines trüben Plasmas ist in Abbildung 1 dargestellt. Es zeigt sich deutlich die Ubberlagerung der trübungs-bedingten Extinktion $\left(\mathrm{E}_{\mathrm{app}}\right)$ durch Plasma-Farbstoffe. Der Verlauf von $E_{\text {app }}$ scheint grob demjenigen zu entsprechen, wie er bei den ModellSuspensionen beobachtet wurde (Abb. 2).

\section{Beqiebung zwiscben Konzentration und $\mathrm{b}$}

Aufgrund der Modell-Versuche wäre zu erwarten, daß zwischen der Konzentration der Partikel und dem Regressions-Koeffizienten keine Beziehung besteht. Um dies zu überprüfen, wurde aus einem Pool von trüben Plasma-Proben eine Verdünnungsreihe hergestellt. Wie aus Tabelle 6 und Abbildung 9 hervorgeht, besteht tatsächlich keine nachweisbare Beziehung zwischen Partikel-Konzentration und $b$.

\section{Beziebung zwischen Konzentration und $\mathrm{E}_{\mathrm{app}}$}

Wurden die gleichen Proben bei konstanter Schichtdicke aber variabler Konzentration und Wellenlänge ausgemessen, so ergab sich, wie in Abbildung 10 dargestellt, eine deutliche Beziehung zwischen Konzentration und $E_{\text {app }}$. Die Beziehung ist dabei nicht analog dem LAMBERT-BEER'schen Gesetz, sondern die einzelnen Kurven weichen gegen die Abszisse ab.

Tab. 6

Regressionskoeffizienten $b$ und Korrelationkoeffizienten $r$ von Proben einer Plasmaverdünnungsreihe; Messungen im Wellenlängenbereich von $350-500 \mathrm{~nm}$

\begin{tabular}{ccc}
\hline $\begin{array}{c}0,154 \mathrm{~mol} / \mathrm{l} \\
\mathrm{NaCl} \\
{[\text { Vol.\%] } \%}\end{array}$ & $\mathrm{b}$ & $\mathrm{r}$ \\
\hline 50 & $-3,14$ & 0,999 \\
40 & $-3,10$ & 0,999 \\
30 & $-3,01$ & 0,999 \\
20 & $-2,91$ & 0,999 \\
10 & $-2,97$ & 0,999 \\
0 & $-2,90$ & 0,998 \\
\hline
\end{tabular}

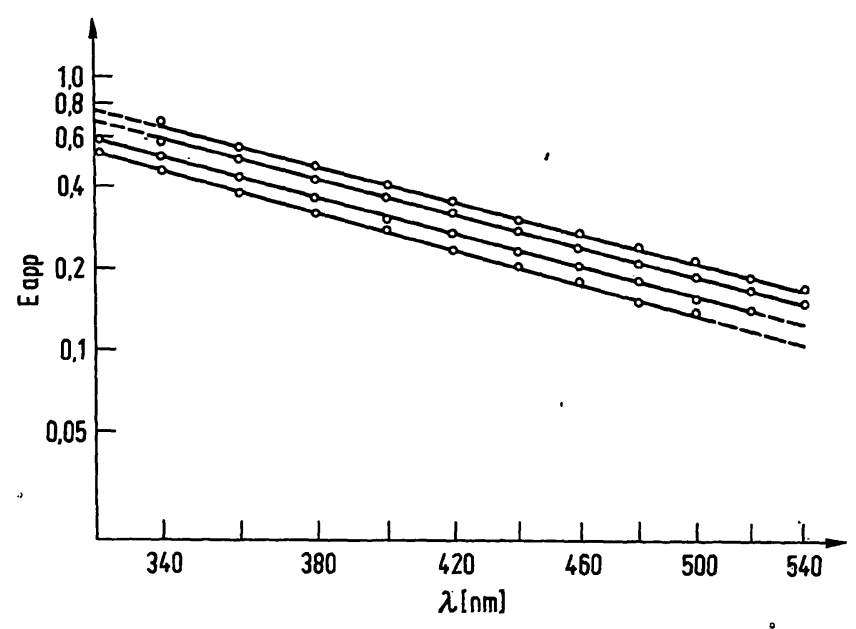

Abb. 9

Beziehung zwischen $E_{a p p}$ und der Wellenlänge bei verschieden verdünnten Lösungen aus einem Pool von trüben Plasma-Proben

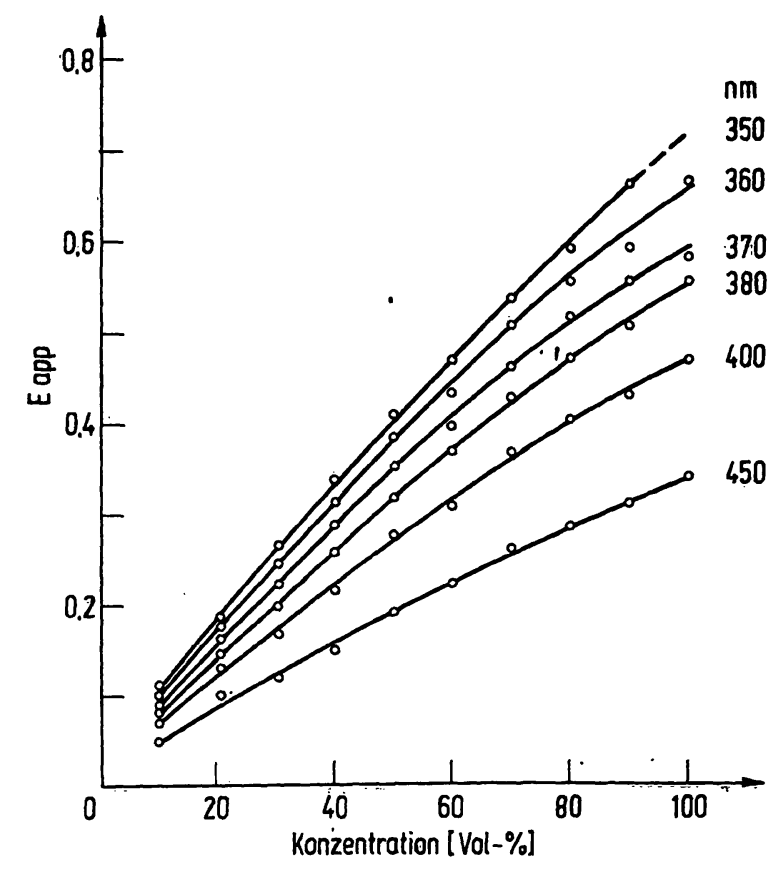

Abb. 10

Beziehung zwischen $E_{a p p}$, der Konzentration und der Wellenlänge von Verdünnungen aus einem Pool von trüben Plasma-Proben

\section{Regressions-Koeffizienten unverdünnter Proben}

In Tabelle 7 und 8 sind die Regressions-Koeffizienten von insgesamt 40 unausgewählten, trüben, unverdünnten Plasma-Proben zusammengestellt. Möglicherweise liegt eine Normal-Verteilung vor (Abb. 11). Die Regressions-Koeffizienten $z$ wischen 2,6 und 4,2 liegen in der Größenordnung von Partikeln (vgl. Abb. 4) von $0,2-0,4 \mu \mathrm{m}$. Es scheint jedoch wahrscheinlicher, daß hier ein Gemisch verschieden großer Teilchen vorliegt, das sich ja, wie die oben erwähnten Versuche mit Mehrkomponenten-Systemen zeigten, analog verhält.

Tab. 7

Regressionskoeffizienten $b$ und Korrelationskoeffizienten $r$ von Plasma-Proben nicht-nüchterner Patienten; Plasmaproben' der 1. Gruppe (s. Methoden)

\begin{tabular}{ccc}
\hline b & r \\
\hline$-3,64$ & 0,998 \\
\hline$-3,32$ & 0,998 \\
$-2,99$ & 0,999 \\
$-3,45$ & 0,999 \\
$-4,60$ & 0,997 \\
$-3,83$ & 0,999 \\
$-4,06$ & 0,998 \\
$-2,48$ & 0,999 \\
$-3,57$ & 0,999 \\
$-3,87$ & 0,999 \\
$-3,20$ & 0,999 \\
$-3,48$ & 0,999 \\
$-3,35$ & 0,999 \\
$-3,53$ & 0,999 \\
$-3,56$ & 0,999 \\
$-4,63$ & 0,999 \\
$-3,23$ & 0,999 \\
$-2,84$ & 0,995 \\
$-1,81$ & 0,998 \\
\hline
\end{tabular}




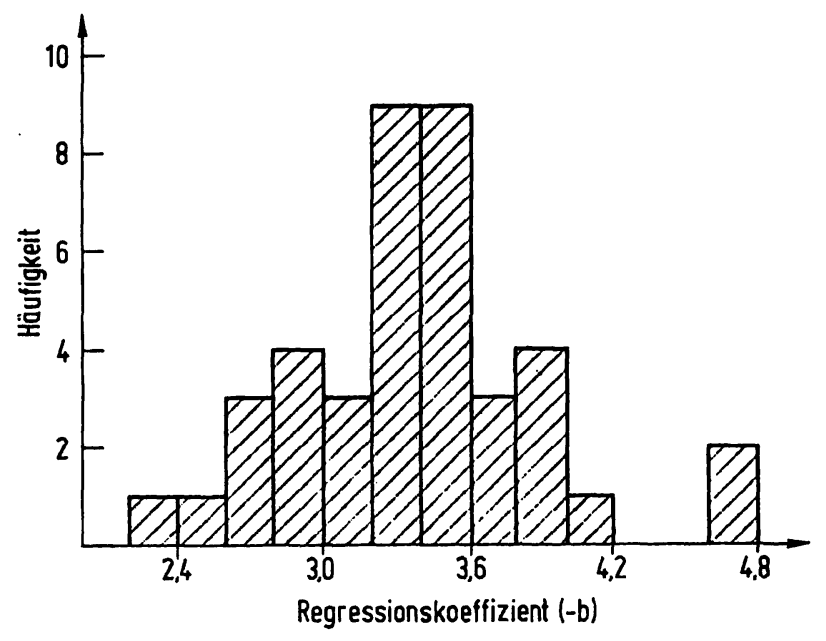

Abb. 11

Häufigkeitsverteilung der Regressionskoeffizienten $b$ aus den $\mathrm{Tab} .7$ und 8

Tab. 8

Regressionskoeffizienten $b$ und Korrelationskoffizienten $r$ von PlasmaProben nicht-nüchterner Patienten; Plasma-Proben der 2. Gruppe (s. Methoden)

\begin{tabular}{cc}
\hline $\mathrm{b}$ & $\mathrm{r}$ \\
\hline$-3,28$ & 0,999 \\
$-3,77$ & 0,998 \\
$-2,63$ & 0,999 \\
$-3,36$ & 0,999 \\
$-3,73$ & 0,998 \\
$-3,05$ & 0,996 \\
$-3,31$ & 0,998 \\
$-3,41$ & 0,999 \\
$-3,46$ & 0,999 \\
$-3,20$ & 0,999 \\
$-2,86$ & 0,998 \\
$-2,37$ & 0,998 \\
$-3,46$ & 0,998 \\
$-3,12$ & 0,998 \\
$-3,52$ & 0,999 \\
$-3,83$ & 0,999 \\
$-3,27$ & 0,999 \\
$-2,62$ & 0,999 \\
$-3,19$ & 0,999 \\
$-2,66$ & 0,999 \\
\hline
\end{tabular}

\section{Diskussion}

Die vorliegenden Untersuchungen haben ergeben, daß sich der trübungsbedingte Extinktionswert $E_{\text {app }}$ von Modell-Suspensionen und trüben Plasma-Proben durch die folgende Gleichung beschreiben läßt:

$$
\log \mathrm{E}_{\mathrm{app}}=-\mathrm{b} \log \lambda+\mathrm{C} \text {. }
$$

Durch die von uns gewählte Meß-Anordnung (Messung in Nah- und Fern-Stellung und Ermittlung der Extinktions-Differenz) kann die durch die Eigen-Farbe des Plasmas bedingte Extinktion weitgehend eliminiert werden. An Modell-Versuchen konnten folgende Beobachtungen gemacht werden:

1. b ist primär eine Funktion der Partikelgröße,

2. auch bei Mehrkomponenten-Systemen charakterisiert b das Mischungs-Verhältnis verschieden großer Partikel, gibt also ein "pseudo-arithmetisches Mittel“" der Partikel-Größe und
3. $\mathrm{E}_{\mathrm{app}}$ ist primär eine Funktion der Partikel-Konzentration.

Die Ermittlung von Regressions-Geraden bei PlasmaProben sollte ermöglichen, sich ein grobes Bild der Partikel-Größe und Partikel-Konzentration zu machen. Es wird nun von Interesse sein, diese Erkenntnisse auf pathologisches Material, vor allem auf Proben von Patienten mit primären Hyperlipidämien auszudehnen.

\section{Anhang (9-12)}

Von Streuung spricht man, wenn bei einer Teilchen-Größe $\mathrm{d} \leqq \lambda$ die Intensitätsanteile von Reflexion, Brechung und Beugung sich nicht mehr trennen lassen. Einfachstreuung liegt vor, wenn Teilchen bei einem mittleren gegenseitigen Abstand vom doppelten Durchmesser $(\AA=2 d)$ unabhängig voneinander streuen. Die Winkelverteilung der Strahlungsdichte bei Einfachstreuung ist abhängig von der Größe, Gestalt und Polarisierbarkeit der Teilchen, ferner von der Beobachtungstichtung. Man unterscheidet:

\section{RAYLEIGH-Streunng}

Diese Theorie der TrNDALL-Streuung befaßt sich mit der Einfachstreuung an Molekülen, deren Durchmesser klein sind gegenüber der Wellenlänge $\lambda: d<0,2 \lambda$ (inkohärente Streustrahlung, $d . h$. die von den streuenden Partikeln ausgehenden Sekundärwellen sind nicht interferenzfähig):

$$
I_{s}=f \frac{N V^{2}}{r^{2} \lambda^{4}} I_{0}
$$

wobei

$\mathrm{I}_{\mathrm{s}}=$ Intensität des gestreuten Lichtes,

$\mathrm{N}=$ Zahl der Kolloidteilchen pro Volum-Einheit,

$\mathrm{V}=$ Volumen des einzelnen Tcilchens,

$\mathrm{I}_{0}=$ eingestrahlte Lichtintensität,

$r=$ Entfernung vom beleuchteten Volum-Element,

$\lambda=$ Wellenlänge,

f = Proportionalitäts-Faktor: Funktion des Winkels zwischen Primärstrahl und Beobachtungsrichtung des gestreuten Lichtes.

Aus dieser Gleichung geht hervor, daß

a) die Intensität der Streustrahlung bei konstanter Teilchengröße proportional der Konzentration (Zahl der Teilchen) ist, und b) die Streulichtintensität unter sonst konstanten Bedingungen proportional der vierten Potenz der Wellenlänge $\lambda$ ist.

\section{Streutung an größeren isotropen kugelförmigen Teilchen}

Für diese Form der Einfachstreuung an isotropen kugelförmigen Teilchen beliebiger Größe ( $z$. B. $d \geqq \lambda / 10)$ existiert die MreTheorie (kohärente Streustrahlung, $\mathrm{d}$. h. die Sekundärwellen werden interferenzfähig, was zu einer partiellen Auslöschung der Sekundärwellen und damit zu einer Abnahme der Gesamtstreulichtintensität führt. Dabei nimmt der Exponent $\mathrm{n}$, der in der RaYLEIGH-Gleichung für die $\lambda^{-n}$-Abhängigkeit der Streuung 4 ist, mit zunehmender Teilchengröße ab und nähert sich dem Wert 0 für sehr große Teilchen. Diese Abhängigkeit des Exponenten n von dem Teilchendurchmesser kann dazu benutzt werden, bei konstanter Wellenlänge $\lambda$ dic Teilchengröße zu bestimmen.

\section{Mehrfachstreuung}

Sobald bei mehreren Teilchen der mittlere gegenseitige Abstand $\overline{\mathrm{A}}<2 \mathrm{~d}$ wird, findet der Ubergang in Mehrfachstreuung statt: die Lichtwelle wird vor dem Verlassen des Mediums mehrmals gestreut. Hicr weist sowohl die remittierte wic die eventuell durchgelassene Strahlung isotrope Streuverteilung auf, wenn die Streudichte (Tcilchenzahl und Schichtdicke) genügend groß sind. Es sei am Rande vermerkt, daß die Streulichtintensität keine spezifische Eigenschaft der streuenden Substanz ist. 
Streulichtmessung (Nephelometrie oder Tyndallometrie): Messung des emittierten TrNDALL-Lichtes.

Für Konzentrationsbestimmungen wird die Intensität des gestreuten Lichtes der Analysenlösung mit derjenigen einer Standardtrübungslösung verglichen. Das Streulicht wird in einem gewöhnlich rechten Winkel zur Primärstrahlung gemessen. Die Konstanz der Teilchengröße, Teilchenform und Polarisierbarkeit wird vorausgesetzt.

Trübungsmessung (Turbidimetrie): Messung der Intensitätsschwächung des primär eingestrahlten Lichtes: scheinbare Extinktion. Für einen bestimmten Konzentrations- und Schicht- dickenbereich gilt eine dem LAMḄ̆RT-BEER'schen Gesetz analoge Beziehung:

$$
E^{*}=\varepsilon^{*} c \mathrm{~d} \text {, wobei }
$$

$E^{*}=$ scheinbare Extinktion, $\varepsilon^{*}=$ scheinbarer Extinktionskoeffizient, auch ,Trübung genannt.

\section{Danksagung}

Die vorliegende Arbeit wurde z.'T. durch den Schweiz. Nationalfond zur Förderung der wissenschaftlichen, Forschung (Projekt Nx. 319669) ermöglicht.

\section{Literatur}

1. Soini, R., Dauwalder, H. \& Richterich, R. (1969), Schweiz. Med. Wochenschr. 99, 1784-1789. - 2. GAEBLER, O. H. (1943), J. Biol. Chem. 149, 251-254. - 3. FoG, J. (1952), Analyst 77 454-460. - 4. Nielsen, M. H. \& Nielsen, N. C. (1962), Scand. J. Clin. Lab. Invest. 14, 605-617. - 5. NIELSEN, N. C. (1963), Scand. J. Clin. Lab. Invest. 15, 613-621. - 6. Nielsen, N. C. (1963), Scand. J. Clin. Lab. Invest. 15, 610-612. - 7. Strutr, J. W. (Lord RAYLEIGH): (1871), Phil. Mag. 41, 107, 274, 447. 8. SAUer, H. (1931), Z. Techn. Physik 12, 148-162. -
9. KoRTüM, G. (1962), Kolorimetrie, Photometrie und Spektrometrie, 4. Aufl. S. 324-345, Springer-Verlag, Berlin. - 10. KorTüM, G. (1969), Reflexionsspektroskopie, 1. Aufl. S. 74 bis 105, Springer-Verlag, Berlin. - 11. Franke, R. \& ThIELE, K. (1969), Physikalisch-chemische Methoden im klinischen Laboratorium, Band II, S. 64-74, VEB Verlag Volk und Gesundheit, Berlin. - 12. KLeIN-WISENBerg, A. v. (1969), Z. Anal. Chem. 247, 166-171.
Prof. Dr. Richterich Chemisches Zentrallabor Inselspital CH-3008 Bern 